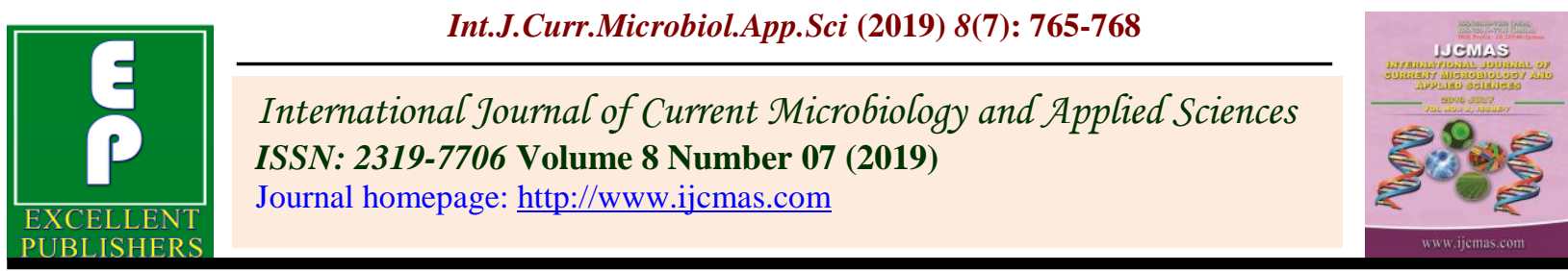

\title{
Pulmonary Manifestations in HIV Patients
}

\author{
Arati Kalakutakar ${ }^{1 *}$, H. Suresh ${ }^{2}$ and Sumant Balagandi ${ }^{1}$ \\ ${ }^{1}$ Department of Microbiology, ${ }^{2}$ Department of Cardiology, KIMS Hubli, India \\ *Corresponding author
}

\section{Keywords}

Pulmonary, Infection, HIV

Article Info

Accepted:

07 June 2019

Available Online:

10 July 2019

\section{A B S T R A C T}

Pulmonary system is the most commonly involved system in people living with HIV as lungs are continuously exposed to the infection as they are rich in macrophages, dendritic cells, lymphocytes etc. In PLHIV immunity is suppressed, hence lungs are prone for infection and non infectious pulmonary diseases. According to autopsy findings, in HIV lung was affected almost upto $100 \%$ in the period of epidemic to $70 \%$ in the HAART era. Pneumonia is most common pulmonary manifestation followed by pulmonary tuberculosis and PCP. Other manifestations are infections like mycobacterium avium complex, fungal infection, nonspecific interstitial pneumonitis, Kaposi sarcoma, lymphoma. Encapsulated organisms like streptococci, $H$. influenzae are responsible for most cases of pneumonia. PCP is the hallmark of AIDS. It is most common with CD4 count $<200 / \mu 1$. About $1 / 3^{\text {rd }}$ of AIDS related deaths are because of tuberculosis. About $60-80 \%$ of HIV patients infected with TB have pulmonary involvement. The main aim of this study is pulmonary involvement in people living with HIV and also to study the co-relation between CD4 count and pulmonary disease in HIV. This is an observational study conducted at KIMS, Hubli. Patients of HIV aged $>18$ yr with pulmonary symptoms were included in the study where as PLHIV with URTI were excluded. A valid written informed consent was taken. Detailed clinical history taking and examination was done. They were subjected to various investigations like CBC, RFT, LFT, CHEST XRAY, CD4 COUNT, SPUTUM ANALYSIS and other investigations like pleural fluid analysis ,CT thorax if needed. Data is statistically analysed. Out of the 36 patients studied 26 were males, 10 female. Majority of them belonged to the group of age 30-50 yr. 24 had CD4 count below 200/ $\mu 1,6$ had CD4 count in the range of 200-500/ $\mu$ l, where as $6 \mathrm{had}>500 / \mu 1$. Most of them were on TLE regimen. 16 had pulmonary Koch, 12 had pneumonia and synpneumonic effusion, 4 had PCP, 2 had bronchiectasis, 2 had bronchitis. All PCP patients had cd4 $<100$.

\section{Introduction}

Pulmonary system is the most commonly involved system in people living with HIV as lungs are continuously exposed to the infection as they are rich in macrophages, dendritic cells, lymphocytes etc. In people living with HIV, immunity is suppressed, hence lungs are prone for infection and noninfectious pulmonary diseases. According to the autopsy findings, in HIV lung was affected almost upto $100 \%$ in the period of epidemic to $70 \%$ in the HAART era.

Pneumonia is most common pulmonary manifestation followed by pulmonary 
tuberculosis and PCP. Bacterial lung infections occur in 5-30\% of HIV positive patients. Patients with HIV are particularly prone to infections by encapsulated organisms like $S$. pneumoniae, $H$. influenzae. Patients with untreated HIV infection have six-fold increase in incidence of pneumococcal pneumonia and 100 fold increase in the incidence of pneumococcal bacteremia.

PCP is the hallmark of AIDS. It is most common with CD4 count $<200 / \mu 1$. The classic finding of a dense perihilar infiltrate is unusual in patients with AIDS.

About $1 / 3^{\text {rd }}$ of AIDS related deaths are because of tuberculosis. About $60-80 \%$ of HIV patients infected with TB have pulmonary involvement. Untreated TB can accelerate the course of HIV infection. Some patients with advanced HIV infection and active TB may have no symptoms of illness, thus screening for TB should be part of the initial evaluation of every patient with HIV infection. HIV infected individuals with skin test reaction of $>5 \mathrm{~mm}$, those with a positive IFN-Y release assay, those who are close household contacts of persons with active TB should receive treatment with 9 months of isoniazid and pyridoxine.

HIV related pulmonary artery HTN is seen in $0.5 \%$ of HIV infected individuals.

Other manifestations are infections like mycobacterium avium complex, fungal infections like Histoplasmosis aspergillosis, nonspecific interstitial pneumonitis, Kaposi sarcoma, lymphoma etc.

The main aim of this study includes, to study the pulmonary involvement in people living with HIV and also to study the co-relation between CD4 count and pulmonary diseases in HIV.

\section{Materials and Methods}

This is an observational study conducted at KIMS, Hubli.

36 HIV patients admitted in KIMS Hubli with pulmonary manifestations were studied.

A valid informed written consent was taken from the subjects.

\section{Inclusion criteria}

HIV patients aged $>18$ yr with pulmonary symptoms

\section{Exclusion criteria}

Patients with upper respiratory tract infection

Those who did not give consent for study.

Detailed clinical history taking and examination was done

They were subjected to various investigations like 1. Complete blood count

RFT

LFT

CHEST XRAY

ECG

2D ECHO

CD4 COUNT

Serum LDH

Sputum Analysis

Other investigations like pleural fluid analysis, CT

Thorax if needed.

Data was entered in Microsoft excel and statistically analysed.

\section{Results and Discussion}

Out of the 36 patients studied 26 were males, 10 female. Majority of them belonged to the 
group of age 30-50 yr. (24). In this study, 14 had CD4 count $<100 / \mu 1,10$ had in between $100-200 / \mu 1,12$ had CD4>200/ $\mu 1$.

Most of them were on TLE regimen. (30), 2 were on ZLN, 2 on TLA.

Out of 36 patients 18 had oral candidiasis.

On complete blood count, 12 had microcytic hypochromic anemia, 8 had normocytic normochromic anemia,

6 had pancytopenia, 4 had dimorphic anemia, 2 had macrocytic anemia.
2 patients had pulmonary artery hypertension on echocardiography.

16 had pulmonary Koch, 12 had pneumonia +/-synneumonic effusion, 4 had PCP, 2 had bronchiectasis, 2 had bronchitis.

All PCP patients had CD4 count $<100 / \mu 1$.

Pulmonary system is the most commonly involved system in people living with HIV. In HIV patients, decreased immunity makes the patients prone for infection. Low CD4 count is responsible for opportunistic infections in people living with HIV.

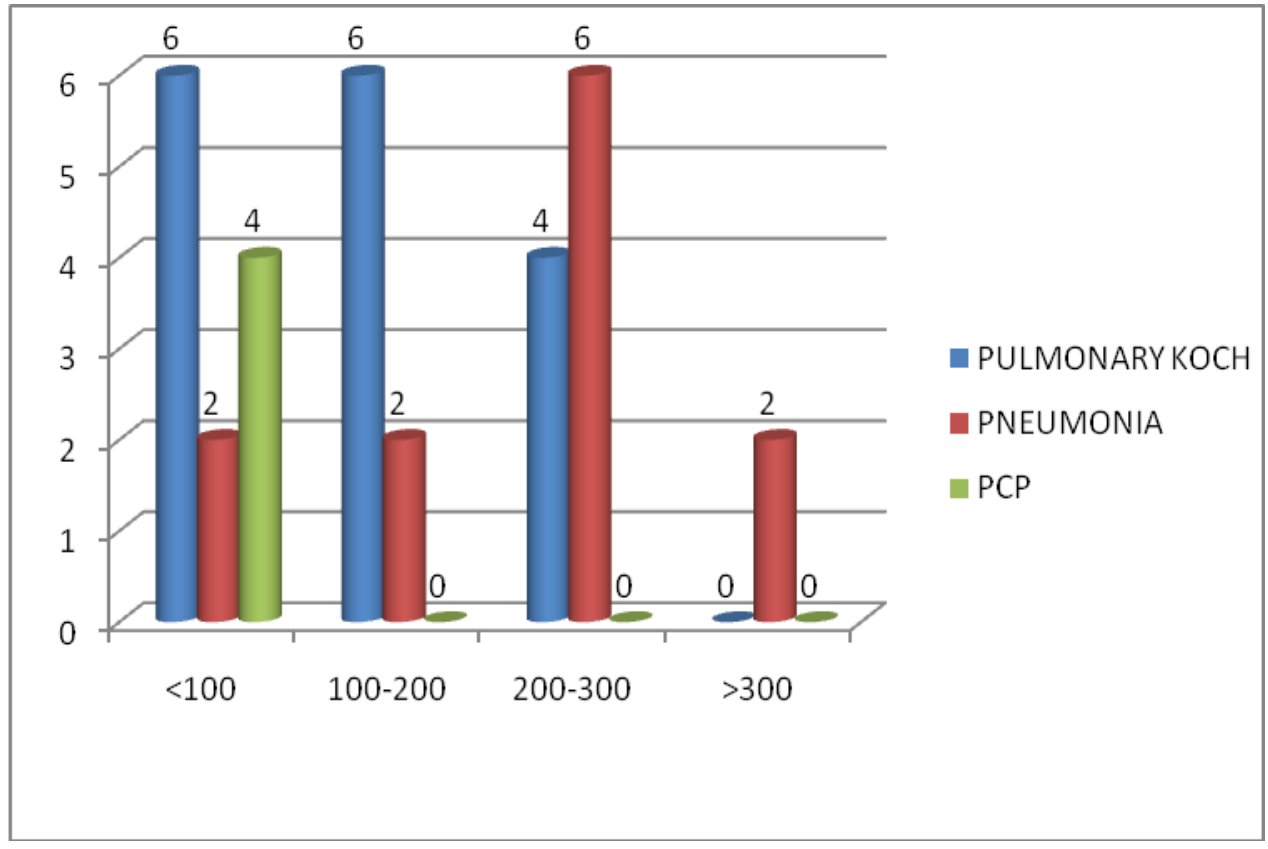

In the present study, pulmonary Koch is the most common pulmonary opportunistic pulmonary involvement (44\%), followed by bacterial pneumonia (33\%), followed by PCP $(11 \%)$. These findings are similar to findings found in many Indian studies like the study conducted by Asmita Mehta et al., in which $72 \%$ had pulmonary Koch, $22 \%$ had bacterial pneumonia, $6 \%$ had PCP. So, this points out that in India still pulmonary Koch is the m.c. infection unlike the western countries where bacterial pneumonia predominate.
CD4 T lymphocyte count acts as an explicit biomarker that provides the information about immune status of the patient. In this study, we found that as the CD4 count decreases, the prevalence of pulmonary Koch increased. The prevalence of pulmonary Koch was max in the people with CD4count $<200 / \mu 1$. In this present study, the majority of bacterial pneumonia belonged to the group of CD4 count 200-300/ $\mu 1$. And all the PCP had CD4 count $<100 / \mu \mathrm{l}$ which is similar to other studies. 
Consolidation and infiltration was the most common chest xray finding in pulmonary Koch (46\%), followed by fibrocavitatory lesions $(40 \%)$, pleural effusion was found in $10 \%$, military mottling in $4 \%$.

Pulmonary Koch was the most common opportunistic pulmonary involvement followed by bacterial pneumonia followed by PCP in people living with HIV.

As the CD4 count decreases, the prevalence of opportunistic infections increase. Prevalence of pulmonary Koch was highest in people with CD4 count $<200 / \mu 1$.

Prevalence of bacterial pneumonia was highest in the group of CD4 count 200$300 / \mu 1$.

Prevalence of PCP was highest in people with CD4 count $<100 / \mu 1$. So PCP should be considered in case of lung infiltrates in patients with CD4 $<100 / \mu 1$.

Consolidation and infiltration was the m.c. chest $\mathrm{x}$ ray finding in pulmonary Koch.

\section{References}

Harrison's principles of Internal medicine $20^{\text {th }}$ edition.

Kristina et al., HIV associated lung infections and complications in the era of combination antiretroviaral therapy. Proceedings of American thoracic society; vol 8, 2011

Mehta a et al.," clinic epidemiological profile of HIV patient with respiratory infection and tuberculosis in western India". Journal of clinical and diagnostic research 2011.

Pu-xuan et al., "correlation between PCP, cd4 count, plasma viral load: quant imaging med surg 2012; 2: 124-129.

Shubhangi V Dhadke, Vithal Dhadke et al., Pulmonary involvement in people living with HIV. Journal of Association of Physicians of India; vol 67-jan 2019.

Toshniwal et al., 'respiratory complications in AIDS seropositive patient in co-relation to CD4 count: an observational crosssectional study; international journal of scientific study $2014 ; 2: 1-5$

\section{How to cite this article:}

Arati Kalakutakar, H. Suresh and Sumant Balagandi. 2019. Pulmonary Manifestations in HIV Patients. Int.J.Curr.Microbiol.App.Sci. 8(07): 765-768.

doi: https://doi.org/10.20546/ijcmas.2019.807.092 